\title{
Comparative RNA-Sequence Transcriptome Analysis of Phenolic Acid Metabolism in Salvia miltiorrhiza, a Traditional Chinese Medicine Model Plant
}

\author{
Zhenqiao Song, ${ }^{1,2}$ Linlin Guo, ${ }^{1}$ Tian Liu, ${ }^{1}$ Caicai Lin, ${ }^{2}$ Jianhua Wang, ${ }^{1,2}$ and Xingfeng Li ${ }^{1,2}$ \\ ${ }^{1}$ Agronomy College, Shandong Agricultural University, Tai'an, Shandong 271028, China \\ ${ }^{2}$ State Key Laboratory of Crop Biology, Agronomy College, Shandong Agricultural University, Tai'an, Shandong 271018, China
}

Correspondence should be addressed to Xingfeng Li; lixf@sdau.edu.cn

Received 1 July 2016; Revised 26 October 2016; Accepted 27 November 2016; Published 17 January 2017

Academic Editor: Mohamed Salem

Copyright (C) 2017 Zhenqiao Song et al. This is an open access article distributed under the Creative Commons Attribution License, which permits unrestricted use, distribution, and reproduction in any medium, provided the original work is properly cited.

\begin{abstract}
Salvia miltiorrhiza Bunge is an important traditional Chinese medicine (TCM). In this study, two S. miltiorrhiza genotypes (BH18 and ZH23) with different phenolic acid concentrations were used for de novo RNA sequencing (RNA-seq). A total of 170,787 transcripts and 56,216 unigenes were obtained. There were 670 differentially expressed genes (DEGs) identified between BH18 and $\mathrm{ZH} 23,250$ of which were upregulated in $\mathrm{ZH} 23$, with genes involved in the phenylpropanoid biosynthesis pathway being the most upregulated genes. Nine genes involved in the lignin biosynthesis pathway were upregulated in BH18 and thus result in higher lignin content in BH18. However, expression profiles of most genes involved in the core common upstream phenylpropanoid biosynthesis pathway were higher in $\mathrm{ZH} 23$ than that in BH18. These results indicated that genes involved in the core common upstream phenylpropanoid biosynthesis pathway might play an important role in downstream secondary metabolism and demonstrated that lignin biosynthesis was a putative partially competing pathway with phenolic acid biosynthesis. The results of this study expanded our understanding of the regulation of phenolic acid biosynthesis in S. miltiorrhiza.
\end{abstract}

\section{Introduction}

Salvia miltiorrhiza Bunge is a widely used traditional Chinese medicine (TCM) with multiple clinical and pharmacological effects associated with cardiovascular, cerebrovascular, and hyperlipidemial diseases [1]. Meanwhile S. miltiorrhiza is also an important model plant for TCM studies due to its short life cycle, small genome size, undemanding growth requirements, and significant medicinal value [2]. The major bioactive components of $S$. miltiorrhiza are diterpenoids, flavonoids, sterols, and water-soluble phenolic acids [3].

The water-soluble phenolic acids in S. miltiorrhiza primarily contain salvianolic acid B (Sal B), rosmarinic acid (RA), caffeic acid, Danshensu, 4-coumaric acid, and tcinnamic acid. Sal B is prevalent in water-soluble extracts and exhibits abundant cardioprotective effects. Thus, Sal B is used as a chemical marker of $S$. miltiorrhiza roots and acts as a phytomedicine. The current Chinese Pharmacopoeia requires the Sal B content in dry S. miltiorrhiza roots higher than 3.0\% (CPA, 2015) [4]. Preliminary investigations have shown that Sal B concentration varies greatly in different genotypes [5-8]. However, the reason for these differences has not yet been investigated to date.

Sal B is presumed to be synthesized from RA [9], and the biosynthetic pathway of RA has been characterized in plants (Figure S1 in the Supporting Information in Supplementary Material available online at https://doi.org/10.1155/2017/ 9364594). RA biosynthesis begins with the aromatic amino acids, L-phenylalanine and L-tyrosine, which are separately converted to the intermediate precursors 4-coumaroylCoA and 4-hydroxyphenyllactic acid through the tyrosinederived pathway and the phenylpropanoid pathway, respectively. These two intermediate precursors are then covalently coupled by several biological reactions to generate RA [10]. A number of key enzymes involved in producing the RAs in S. miltiorrhiza have been identified [11-13], while the detailed pathway from RA to Sal B has not yet been characterized. 


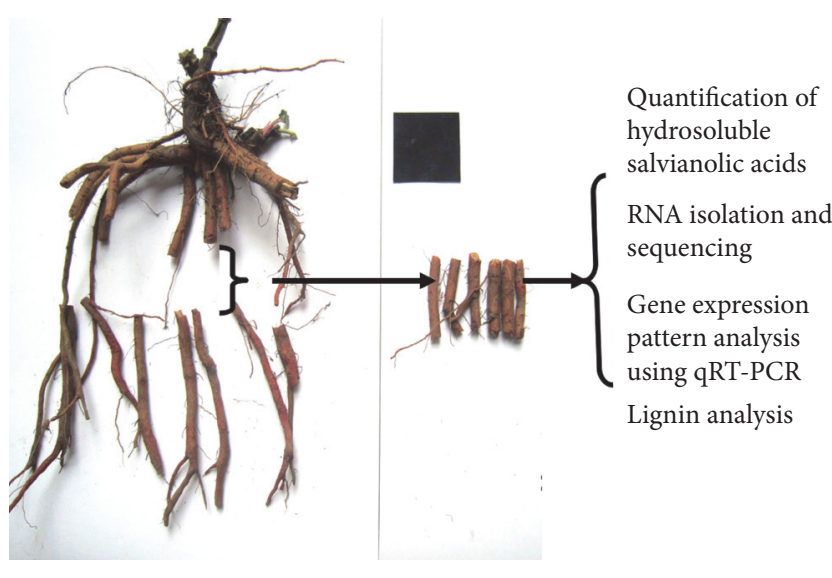

FIGURE 1: The root segments of S. miltiorrhiza for further analysis.

In recent years, several kinds of transcriptome sequencing platforms such as Sanger sequencing, 454 pyrosequencing, and Solexa sequencing have been used for discovering related genes involved in secondary metabolism pathways of $S$. miltiorrhiza. However, previous studies were mainly focused on tanshinones biosynthesis and its regulation [14-18]; few reports were focused on the phenolic acid biosynthesis $[14,16,19]$. Furthermore, those previous RNA-seq studies were focused only on the development stages, tissue types, and hairy root cultures of one specific genotype; studies focused on different genotypes with diverse concentrations of bioactive components have not been conducted.

Compared with forward sequencing methods, Illumina RNA-seq technology has been proved as a more effective approach for transcriptome study, with higher read depth and prediction accuracy capabilities [20]. However, it is seldom used in S. miltiorrhiza transcriptome study.

In this study, two different lines (ZH23 and BH18) were bred through individual selection from the natural germplasm of S. miltiorrhiza; the Sal B content in $\mathrm{ZH} 23$ roots was significantly higher than that in $\mathrm{BH} 18$. In the present study, Illumina RNA-seq technology is used to investigate the transcriptome profile of these two lines, with the goal of unraveling the differentially expressed genes (DEGs) involved in phenolic acid biosynthesis pathway. The results of this study would expand our understanding of the regulation of phenolic acid biosynthesis in S. miltiorrhiza.

\section{Materials and Methods}

2.1. Plant Materials. Two different S. miltiorrhiza lines, BH18 and $\mathrm{ZH} 23$, were used in this study. Each line is asexually propagated through root cutting. Thirty individuals of each genotype were planted in $5 \mathrm{~m}^{2}$ plots and were grown under identical conditions at the Botanical Garden in the Agronomy College of Shandong Agricultural University. Mid-segments roots with a length of $5 \mathrm{~cm}$ were collected from 9 randomly selected individual plants for each line at the harvest stage (October 25th, 2014) and divided into four parts (Figure 1): The first part was mixed with equivalent fresh weight for RNA sequencing. Another part was used for RNA isolation and real-time PCR analysis. All the above samples were frozen immediately in liquid nitrogen and stored at $-80^{\circ} \mathrm{C}$ until further processing. The third part was used to determine phenolic acid content, and the remaining part was used for lignin analysis.

\subsection{Extraction, Analysis, and Quantification of Hydrosoluble} Salvianolic Acids Using High-Performance Liquid Chromatography (HPLC). The collected roots of both genotypes were dried at $30^{\circ} \mathrm{C}$ to constant weights and then ground into powder and $100 \mathrm{mg}$ powder of each line was extracted with $50 \mathrm{~mL}$ methanol in an ultrasonic bath for $1 \mathrm{hr}$. The supernatant was analyzed by HPLC after filtration through a $0.22 \mu \mathrm{m}$ membrane. Each experiment was run in triplicate. The HPLC analyses of the phenolic acids were performed as described by the CPA (2015) [4].

2.3. RNA Isolation and Quality Verification. To eliminate the variation between individual plants, the RNA were isolated from mixed root segments of three individual plants and three RNA samples were extracted for each line. For RNA extraction, the collected roots were homogenized in liquid nitrogen first, and then the total RNA was extracted using TRIzol@ Reagent (Invitrogen, San Diego, USA) and treated with DNaseI (Invitrogen). The quantity and quality of the extracted RNA were verified by gel electrophoresis and a NanoPhotometer ${ }^{\circledR}$ spectrophotometer (IMPLEN, CA, USA).

2.4. cDNA Library Construction and Sequencing. Equivalent quantities of total RNA from three samples were mixed to prepare the pooled RNA samples for RNA-seq. A total quantity of $30 \mu \mathrm{g}$ of mixed RNA was confirmed for RIN values above 8.0 and was used as input material for the construction of RNA-seq library. The library was generated using the Illumina TruSeq ${ }^{\mathrm{TM}}$ RNA Sample Preparation Kit (Illumina, San Diego, USA) following the manufacturer's recommendations. The clustering of the index-coded samples was performed on a cBot Cluster Generation System using the TruSeq PE Cluster Kit v3-cBot-HS (Illumina) according to the manufacturer's instructions. After cluster generation, the library preparations were sequenced on an Illumina Hiseq 2000 platform, and $100 \mathrm{bp}$ paired-end reads were generated.

2.5. Data Processing, Assembly, and Annotation. Clean data (clean reads) were obtained by removing the reads containing adapters, the reads containing poly- $\mathrm{N}$, and low-quality reads, from the raw data. The Q20, Q30, GC-content, and sequence duplication levels of the clean data were calculated. All of the downstream analyses were based on high-quality clean data. The left read files (read1 files) from all of the libraries/samples were pooled into a single left.fq file, and the right read files (read2 files) were pooled into a single right.fq file. The transcriptome assembly was accomplished based on the left.fq and right.fq files, using Trinity [21] with min_kmer_cov set to 2 and all of the other parameters set to default.

All of the assembled unigenes of the two lines were searched against the NCBI Nr database to identify their putative mRNA functions using the BLAST algorithm [22] with 
an $E$-value cut-off of $10^{-5}$. Additionally, Gene Ontology (GO) terms were extracted from the strongest hits obtained from BLASTx against the $\mathrm{Nr}$ database using the Blast2GO program [23]. The BLAST algorithm was also used to align unique sequences to Nt, Pfam, KOG, Swiss-Prot (manually annotated and reviewed protein sequence database), COG (Clusters of Orthologous Groups of proteins), and KO (KEGG Ortholog database) [24] (with an $E$-value cut-off of $10^{-5}$ ) to predict possible functional classifications and molecular pathways.

2.6. Gene Expression Pattern Analysis. Gene expression levels were estimated by mapping clean reads to the Trinity transcripts assembly using RSEM [25] for each sample. The abundance of all of the genes was normalized and calculated using uniquely mapped reads with the RPKM method [26]. A differential expression analysis of the two samples was performed by modeling count data with negative binomial distributions, as described in the DEGseq method [27]. $P$ values were adjusted using $Q$ values [27], and [ $q$ value < 0.005 and $\mid \log _{2}$ (fold change) $\mid>1$ ] was set as the threshold for a significant differential expression level. The identified differentially expressed genes (DEGs) were used for the GO and $\mathrm{KO}$ enrichment analyses. The GO enrichment analyses were performed using GOseq [28], based on the Wallenius noncentral hypergeometric distribution, to map all of the DEGs to terms in the GO database $(P$ value $\leq 0.05)$ to identify significantly enriched GO terms in the DEGs. The KEGG pathway enrichment analysis of the DEGs was completed using KOBAS [29].

2.7. Real-Time Quantitative RT-PCR ( $q R T-P C R)$ Assay. Gene expression levels were determined via qRT-PCR [30]. A housekeeping gene, Ubiquitin, was used as a control to account for the variation in the cDNA template levels. The target and reference cDNAs were amplified with identical reaction mixtures in a single iCycler iQ5 run. Gene-specific primers for quantitative PCR are listed in Table S1 in the Supporting Information.

2.8. Lignin Analysis. The main roots from the harvest stage were used for analyzing the lignin contents of both lines. Free-hand sections were immediately immersed in 5\% phloroglucinol (dissolved in 100\% ethanol) for $2 \mathrm{~min}$ and then incubated in concentrated $\mathrm{HCl}$. The samples were then photographed within $30 \mathrm{~min}$ [31]. The Klason lignins were then extracted and measured according to the protocol of Zhang et al. [32].

\section{Results}

3.1. The Content of Phenolic Acid Component in ZH23 and BH18. The phenolic acid concentrations of $\mathrm{ZH} 23$ and $\mathrm{BH} 18$ roots at the harvest stage were determined via HPLC (Figure 2). Seven common peaks of phenolic acid components were obtained in both lines, in which two peaks were identified as Sal B and RA according to the consistency of retention time and spectra of the peaks (Figure 2). The total peaks area were considered to be the total salvianolic
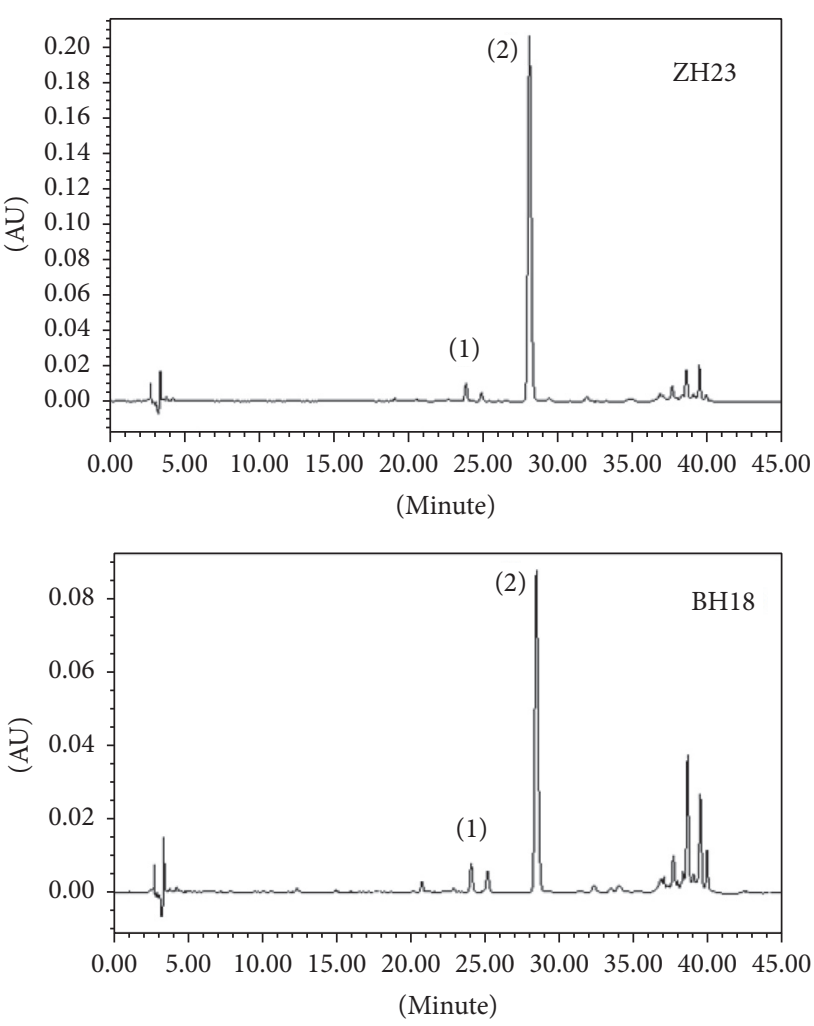

FIGURE 2: Elution profile of $\mathrm{ZH} 23$ and $\mathrm{BH} 18$ on the HPLC-UV chromatograms. The retention times for RA (1) and tanshinone Sal B (2) were $23.901 \mathrm{~min}$ and $28.303 \mathrm{~min}$, respectively.

acid. Results showed that the content of Sal B, RA, and the total phenolic acid in $\mathrm{ZH} 23$ were higher than those of BH18 (Table S2). The ratio of Sal B in total phenolic acid was also higher in $\mathrm{ZH} 23$ (about 75\%) than that in BH18 (60\%). In both lines, Sal B was found to be the main compounds.

3.2. RNA-seq and De Novo Assembly of ZH23 and BH18. Mixed root samples at the harvest stage of $\mathrm{ZH} 23$ and $\mathrm{BH} 18$ were used for RNA-seq analysis. After quality control and the removal of low-quality reads, a total of $81,665,757$ and $61,924,629$ clean reads were attained from BH18 and ZH23 cDNA libraries. More than $90 \%$ of the clean reads had a read quality of Q30 (sequencing error rate $0.1 \%$ ) or higher. Due to the absence of genomic reference sequences, de novo assembly was applied while the clean reads from both lines were pooled together, resulting in 170,787 transcripts and 56,216 unigenes with N50 lengths of 2,026 and 1,577, respectively.

The unigene annotations were performed by BLAST searches $\left(E\right.$-value $\left.\leq 10^{-5}\right)$. A total of 25,921 unigenes $(46.11 \%$ of the unigenes) were annotated with significant BLAST results in the $\mathrm{Nr}$ database; 20,128 unigenes $(36.59 \%$ of the unigenes) were annotated in the Swiss-Prot database; 20,574 unigenes were annotated in Pfam, and 22,424 unigenes were annotated in the GO database. In total, 28,707 unigenes were 
TABLE 1: Gene annotation by searching against public databases.

\begin{tabular}{lcc}
\hline Gene annotation & $\begin{array}{c}\text { Number of } \\
\text { unigenes }\end{array}$ & Percentage (\%) \\
\hline Annotated in NR & 25,921 & 46.11 \\
Annotated in NT & 9,841 & 17.5 \\
Annotated in KO & 9,162 & 16.29 \\
Annotated in & 20,128 & 35.8 \\
Swiss-Prot & 20,574 & 36.59 \\
Annotated in & 22,424 & 39.88 \\
PFAM & 11,950 & 21.25 \\
Annotated in GO & 3,375 & 6 \\
Annotated in KOG & & \\
$\begin{array}{l}\text { Annotated in all } \\
\text { databases }\end{array}$ & 28,707 & 51.06 \\
Annotated in at & 56,215 & \\
least one database & Total unigenes & \\
\hline
\end{tabular}

annotated in at least one database (Table 1). To further identify the active biological pathways in S. miltiorrhiza, 9,162 unigenes annotated by BLAST analysis against the KAAS (KEGG Automatic Annotation Server) were mapped to 250 reference canonical pathways. These canonical pathways were classified into five main categories: "cellular processes," "environmental information processing," "genetic information processing," "metabolism," and "organismal systems" (Figure S2 in the Supporting Information). The pathways with the highest representation were "translation" (937 unigenes, 10.23\%) and "carbohydrate metabolism" (832 unigenes, 9.08\%). These annotations and classifications provided a resource for investigating specific pathways in S. miltiorrhiza, such as the RA biosynthetic pathway. The biosynthetic pathway leading to Sal B and RA is thought to entail both phenylpropanoid and tyrosine-derived pathways. Totally, 96 unigenes were clustered into "phenylpropanoid biosynthesis"; 52 and 51 unigenes were classified into the "tyrosine metabolism" and "phenylalanine, tyrosine, and tryptophan biosynthesis" subpathways, respectively.

3.3. RPKM Density Distribution of Transcript Profiling. The two genotypes showed similar RPKM density distributions, which suggested that their transcription profiles were similar (Figure S3 in the Supporting Information). The top two most abundant transcripts in the roots, which accumulated at 32,104 RPKM, were the S. miltiorrhiza SMLII mRNA, which is consistent with previous transcriptome data using $454 \mathrm{GS}$ FLX from roots grown for 2 years [15].

3.4. DEGs of the Two Genotypes. On the basis of the applied threshold [ $q$-value $<0.001$ and $\log _{2}$ (fold change) $>1$ ], 670 genes (1.19\% of the total genes) were identified as significant differentially expressed genes (DEGs) between these two lines, which comprised 250 upregulated genes (accounting for $37.32 \%$ of the significant DEGs) and 420 downregulated genes (62.68\%) in $\mathrm{ZH} 23$, respectively (Figure 3 ).

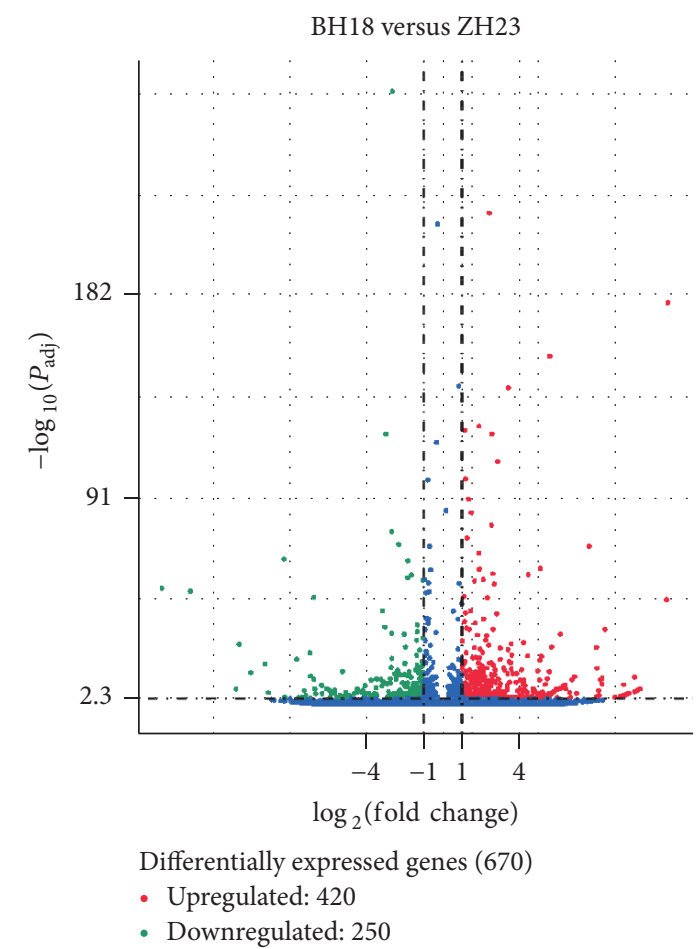

FIGURE 3: Comparison of expression patterns of differential unigenes identified between $\mathrm{BH} 18$ and $\mathrm{ZH} 23$. The red dots represent upregulated DEGs, the green dots represent downregulated DEGs, and the blue dots represent non-DEGs. In total, 670 unigenes were identified as differentially expressed genes $(Q$ value $<$ 0.005 and $\log _{2}$ (fold change) $>1$ ) between $\mathrm{BH} 18$ and $\mathrm{ZH} 23$.

To investigate the biochemical pathways of the DEGs, we mapped the DEGs in terms in the KEGG database and compared these results with the complete transcriptome background. Figure 4 shows a scatterplot of the DEGs that were enriched in the top 20 KEGG pathways. Of these, two pathways, "phenylpropanoid biosynthesis" (Corrected $P$ value 0.00000117 ) and "biosynthesis of secondary metabolites" (Corrected $P$ value 0.000059 ), were the most significantly enriched pathways.

3.5. DEGs Involved in the Phenolic Biosynthesis Pathway. The biosynthetic pathway leading to Sal B and RA is thought to entail both phenylpropanoid and tyrosine-derived pathways. In total, $11 \mathrm{DEG}$ unigenes were related to phenylpropanoid biosynthesis or related pathways. Figure 5 shows a representation of the DEG unigenes related to phenolic acid biosynthesis and the competing pathways.

Among the 11 DEGs, one DEG, comp49431_cl, was upregulated 1.582-fold in $\mathrm{ZH} 23$ and was annotated as "4coumarate:CoA ligase 2 (Sm4CL2, EC 6.2.1.12)," which is involved in the core-phenylpropanoid pathway. Enzyme 4CL plays an important role in the general phenylpropanoid pathway by catalyzing a series of aromatic substrates to form their corresponding hydroxycinnamoyl-CoA esters, which are key precursors for the biosynthesis of numerous 


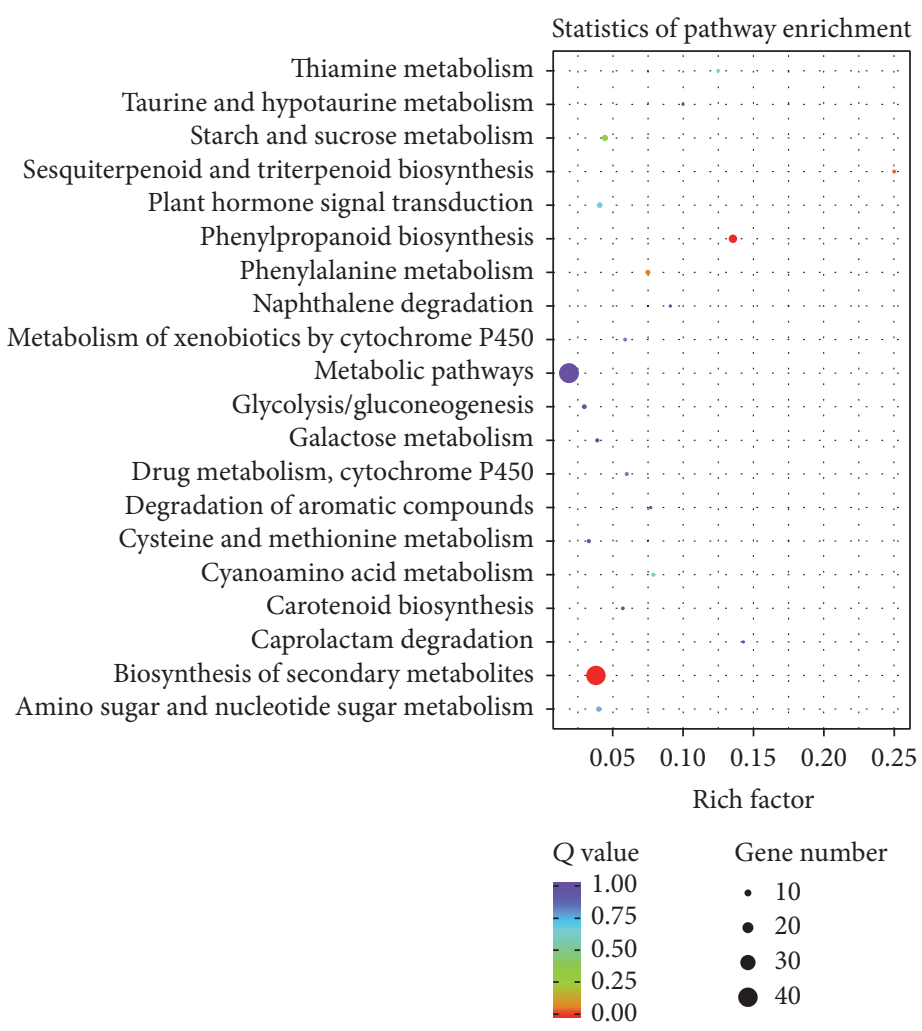

FIGURE 4: Scatterplot of differentially expressed genes enriched in the top 20 KEGG pathways. Rich factor represents the ratio of the number of DEGs and the number of all unigenes in the pathway; $Q$ value represents corrected $P$ value.

phenylpropanoid derivatives, including RA, flavonoids, and lignin [33].

The other DEGs were all downregulated in $\mathrm{ZH} 23$ and upregulated in BH18, and those genes were associated with the branched lignin biosynthesis pathway. Among them, comp50099_cl, which was annotated as shikimate Ohydroxycinnamoyl transferase (HCT, EC: 2.3.1.133), had an expression level in $\mathrm{BH} 18$ that was 2.17-fold higher than $\mathrm{ZH} 23$. HCT catalyzes the synthesis of the shikimate and quinate esters of p-coumaric acid [34], which controls the biosynthesis of two major lignin building units, namely, the guaiacyl (G) and syringyl (S) units. Comp51337_c0 (1.1205), which was annotated as "ferulate-5-hydroxylase (F5H, EC: 1.14.-.-)," a cytochrome P450-dependent monooxygenase (P450) of the general phenylpropanoid pathway [35], was upregulated 1.12-fold in $\mathrm{BH} 18$. F5H catalyzes the hydroxylation of ferulic acid, coniferaldehyde, and coniferyl alcohol in the pathways leading to sinapic acid and the syringyl unit of lignin [36]. Comp24721_c0, which was annotated as "coniferyl-aldehyde dehydrogenase (REF1, EC: 1.2.1.68)," was upregulated 3.527fold and catalyzes coniferyl aldehyde to ferulate acid. Two unigenes, comp48837_c0 (2.1147-fold) and comp42506_c0 (1.2278-fold), were annotated as "cinnamyl-alcohol dehydrogenase (CAD, EC: 1.1.1.195)". CAD specifically catalyzes the reduction of cinnamaldehydes to cinnamyl alcohols during the final step of monolignol biosynthesis. Several homologs of CAD play important roles in the lignification of elongating stems in Arabidopsis [37]. In addition, five unigenes were annotated as "peroxidase (POD, EC: 1.11.1.7)" also upregulated in BH18, including comp23935_c0 (3.361-fold), comp49112_c0 (2.2654-fold), comp24685_c0 (4.1104-fold), comp36960_c0 (1.7491-fold), and comp45749_c0 (4.0349fold). The last major step in lignin synthesis involves monolignol dehydrogenation and polymerization, and different classes of oxidative enzymes are implicated in this step, including class III peroxidase (POD; EC 1.11.1.7), laccase, ascorbate peroxidase, and NADPH oxidase [38].

3.6. The Expression Profiles of Genes Related to RA Biosynthesis. The gene expression patterns of SmPAL, SmC4H, Sm4CL1, Sm4CL2, SmTAT, and SmHPPR, which are reported to be associated with RA biosynthesis, were validated by qRT-PCR. Ubiquitin was used as an internal reference. The transcription levels of these genes were low in both lines compared with the internal reference gene (Figures 6(a)6(b)). The expression levels of SmPAL, SmC4H, Sm4CL1, Sm4CL2, SmTAT, and SmHPPR in ZH23 were all significantly higher than that in BH18, which were generally consistent with the gene expression profiling from the RNA-seq data.

We also used qRT-PCR to investigate the gene expression patterns of 5 DEGs significantly enriched with phenylpropanoid biosynthesis (Figures 6(c)-6(d)). The expression levels of SmHCT (comp50099_c1), SmF5H (comp51337_c0), SmREF1 (comp24721_c0), SmCAD1 (comp48837_c0), and SmPOD (comp36960_c0) in BH18 were all higher than that in $\mathrm{ZH} 23$, which is also consistent with RNA-seq results. 


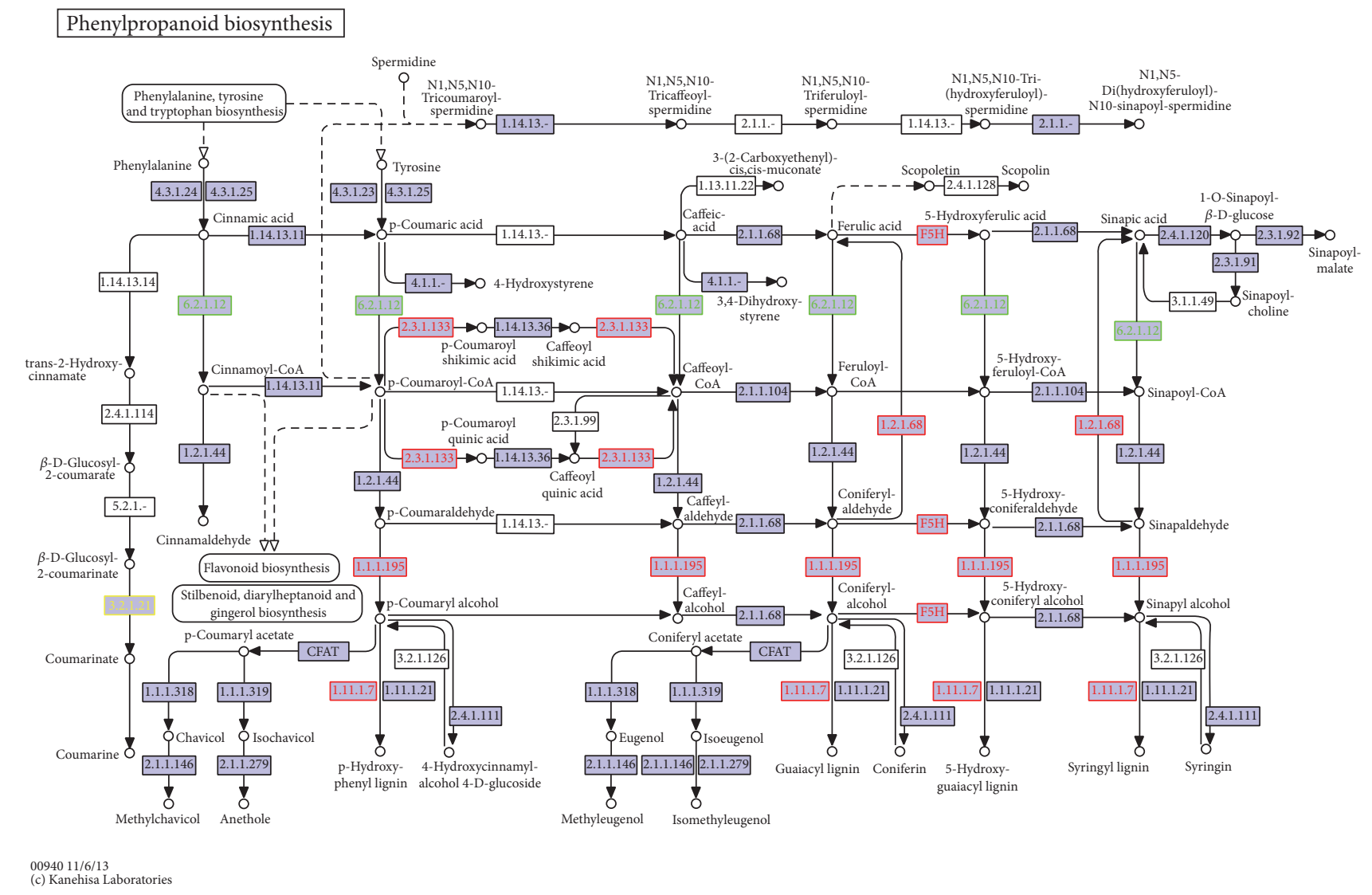

FIGURE 5: Representation of genes related to phenolic acid biosynthesis. Note: the red frames and red letters or numbers represent upregulated DEGs in BH18; the green frames and green letters or numbers represent downregulated DEGs in BH18; the purple frames represent nonDEGs.

\section{Discussion}

4.1. S. miltiorrhiza Transcriptome Study Using RNA-seq. For species without reference genomes, RNA-seq can effectively determine accumulation levels of mRNA that can be associated with gene function, and this may potentially be an underlying cause of phenotypic variation. Previous studies have demonstrated the Sal B concentrations in S. miltiorrhiza vary greatly between different genotypes [5, 7, 8]. Thus, the transcriptome profiling of $S$. miltiorrhiza genotypes with different concentrations of bioactive components will promote a relatively accurate understanding of the molecular mechanisms of secondary metabolism in S. miltiorrhiza, especially for phenolic acids biosynthesis. However, several previous transcriptome investigations of S. miltiorrhiza only used one specific cultivar or a single genotype. In this study, two genotypes with different concentrations of bioactive components were selected to analyze phenolic acids biosynthesis using RNA-seq data.

In RNA-seq, the read lengths and the assembled total lengths are important factors for gene function analysis [20]. In recent years, researchers have undertaken transcriptome studies on S. miltiorrhiza using different RNA-seq methods. Yan et al. [14] selected and assembled, using Sanger sequencing, 10,288 expressed sequence tags (ESTs, with sizes $\geq 100 \mathrm{bp}$ ) into 4,225 unigenes from the pBluescript II XR cDNA library. Li et al. [15] used the 454 GS FLX platform and obtained 46,722 ESTs with an average read length of $414 \mathrm{bp}$, which were assembled into 18,235 unigenes. Hua et al. [16] generated 56,774 unigenes ( $\geq 200 \mathrm{bp}$ ) with an N50 length of $535 \mathrm{bp}$ and a total length of $26.49 \mathrm{Mb}$ by Solexa deep RNA-seq. Using Roche's 454 GS-FLX system, Yang et al. [17] reported a total of 64,139 unigenes with an N50 length of $750 \mathrm{bp}$ and a total size of 26.4 Mb. Gao et al. [18] reported a final total of 20,972 nonredundant genes with a total length of $11.8 \mathrm{Mb}$. Compared with the previous studies, our Illumina sequencing data showed a longer N50 length $(1,577 \mathrm{bp})$ and a larger coverage of the entire genome with a total length of $47.45 \mathrm{Mb}$, which provided a more valuable resource for investigating plant development and the biosynthesis of effective components in S. miltiorrhiza.

4.2. Valuable Clues Regarding the Different Sal B Concentrations in Two S. miltiorrhiza Lines. In addition to the phenolic acid-branched pathway, phenylpropanoid metabolism also transforms phenylalanine into a variety of molecules, including lignins and flavonoids such as flavanones, dihydroflavonols, and anthocyanins. Thus, the phenolic acid, lignin, and flavonoid biosynthetic pathways share a common core phenylpropanoid pathway in their early steps. 


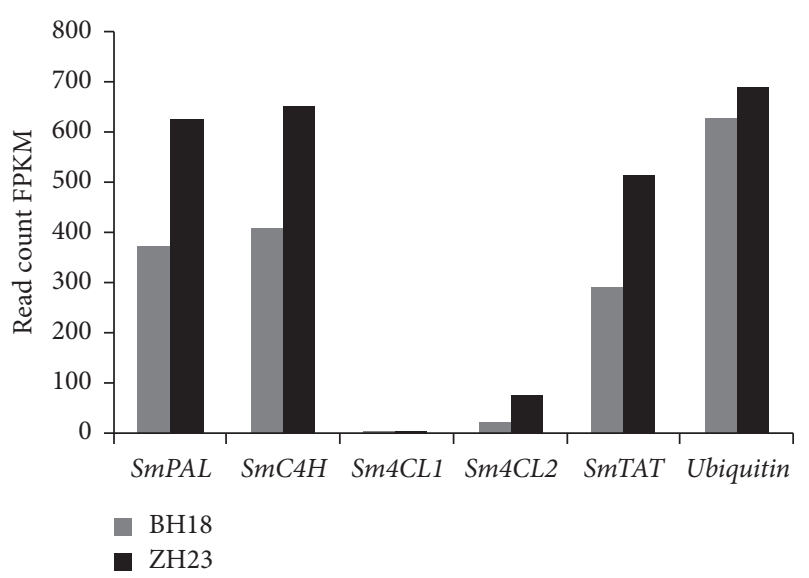

(a)

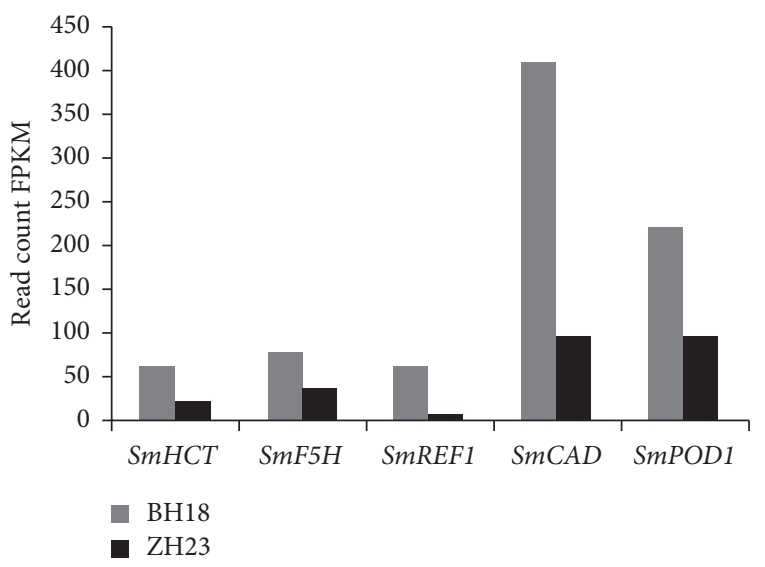

(c)

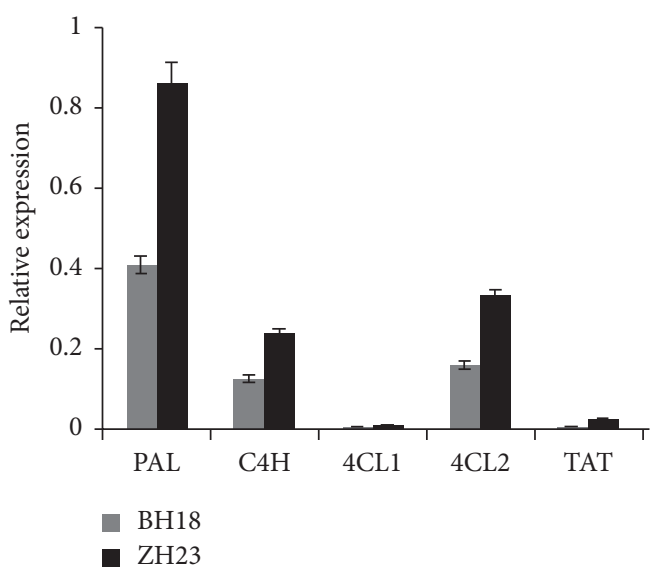

(b)

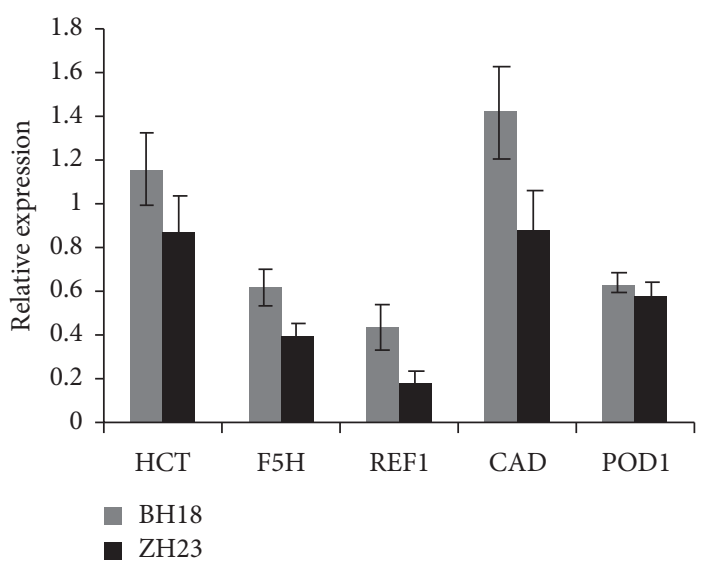

(d)

FIGURE 6: Expression patterns of the genes involved in phenolic acid biosynthesis in BH18 and ZH23 detected by DGE and validated by qPCR. (a) RA biosynthesis genes detected by digital gene expression (DGE). (b) The 5 genes selected from above were confirmed by qPCR. (c) Lignin biosynthesis genes detected by digital gene expression (DGE). (d) The 5 genes related with lignin biosynthesis genes confirmed by qPCR. The results represent the means \pm standard deviations of experiments performed in triplicate.

A total of 25 genes encoding up to 13 enzymes are annotated as being involved in RA biosynthesis in S. miltiorrhiza. Some previously reported genes in S. miltiorrhiza were generally identified in our RNA-seq data, and most of them showed higher expression in root of $\mathrm{ZH} 23$ than that in BH18. A central role gene, $S m 4 C L$, in the core phenylpropanoid pathway, was upregulated 1.52 -fold in $\mathrm{ZH} 23$. In addition, we detected 7 unigenes annotated as "4CL," excluding comp49431_cl, which was annotated as Sm4CL2. Another unigene with relatively low expression levels was annotated as Sm4CL1 (comp50706_c0), with RPKMs of 5.89 in ZH23 and 2.49 in BH18. This difference in expression is consistent with previous reports that suggest that $S m 4 C L 2$ rather than Sm4CL1 might be responsible for Sal B biosynthesis in $S$. miltiorrhiza roots [39]. The remaining five 4CL were not previously reported to be present in S. miltiorrhiza, and one had the highest level of expression, with RPKMs of 121.72 in $\mathrm{BH} 18$ and 146.65 in $\mathrm{ZH} 23$; thus, there was no difference between the two lines, and its length was the longest at 3,608 bp. This finding implies that maybe there are more than two functional 4CLs in the S. miltiorrhiza transcriptome.

As a result of the downregulation of Sm4CL2, and also most of genes in common core phenylpropanoid pathway in $\mathrm{BH} 18$, coumarate-CoA co-precursors might have relatively lower expression patterns, thus "reducing income" for the entire downstream pathway. We considered three mainly specific branch pathways: flavonoids, phenolic acids, and lignin. There were no significant DEGs among 28 background unigenes in the flavonoid pathways. To date, the detailed phenolic acid-branched pathway from RA to Sal B has not been characterized. However, the DEGs involved in the lignin pathway indicated it was probably a significantly enriched alternative pathway. Five key enzyme genes in the lignin pathway, for example, HCT, F5H, CAD, REF1, and POX, were upregulated in $\mathrm{BH} 18$, which maybe increase lignin biosynthesis to a high level, which can be further shown by histochemical properties and lignin concentrations in roots. We found that a greater number of vascular rays and cells per ray were stained in BH18 (Figure 7), and the 


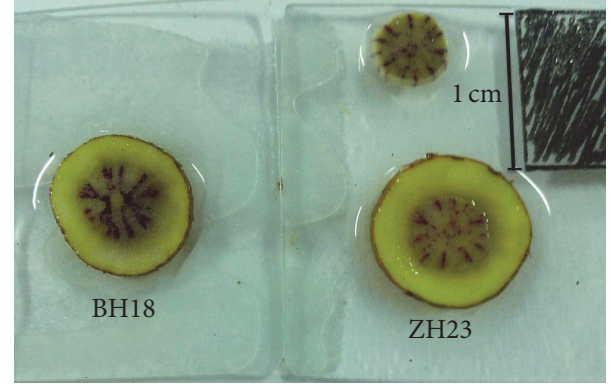

FIGURE 7: Lignin staining of BH18 and ZH23.
DEGs: Differentially expressed genes

QRT-PCR: Real-time quantitative reverse transcriptase polymerase chain reaction

RPKM: Reads per kilobase per million

4CL2: 4-coumarate:CoA ligase 2

CT: $\quad$ Shikimate O-hydroxycinnamoyl transferase

450: $\quad$ Cytochrome P450-dependent monooxygenase

CAD: Cinnamyl-alcohol dehydrogenase

POD: $\quad$ Peroxidase

CCR: Cinnamoyl CoA reductase

AtPAP1: Arabidopsis Production of Anthocyanin Pigment 1 transcription factor

COMT: Caffeic acid O-methyltransferase.

\section{Competing Interests}

Klason lignin concentration in $\mathrm{BH} 18$ was higher compared with $\mathrm{ZH} 23(0.2525 \mathrm{mg} / \mathrm{g}$ versus $0.2085 \mathrm{mg} / \mathrm{g})$. Therefore, increasing lignin biosynthesis can be regarded as "increasing expenditure." We speculated that when compared with BH18, the high Sal B line $\mathrm{ZH} 23$ has a higher number of precursors, signifying a decrease in lignin biosynthesis, which allows metabolic flow to be rerouted toward the accumulation of phenolic acids, which ultimately increases the Sal B concentration. It was reported that transgenic S. miltiorrhiza plants with downregulated cinnamoyl CoA reductase (SmCCR) exhibited dwarfing phenotypes, and both of the $S$ and $G$ lignin monomers were decreased by more than $60 \%$. In contrast, the biosynthesis of phenolic acids, Danshensu, RA, and Sal B, was significantly increased [40]. Zhang et al. [41] also reported that the S lignin concentration decreased approximately 30-40\% with ectopic expression of Arabidopsis Production of Anthocyanin Pigment 1 transcription factor (AtPAP1) via the cosuppression of SmCCR and caffeic acid O-methyltransferase (SmCOMT).

Although these data show that cooverexpression of lignin pathway genes, for example, HCT, CAD, F5H, ERF1, and $\mathrm{POD}$, enhanced the lignin accumulation in $\mathrm{BH} 18$, the lower expression of genes in the core common upstream pathway will finally result in lower Sal B and RA contents in BH18 compared with $\mathrm{ZH} 23$. This result indicated that expression of genes involved in the core common upstream pathway plays a more important role in downstream second metabolism and also obviously indicated the lignin pathway as potential competing pathway with Sal B biosynthesis.

\section{Abbreviations}

TCM: Traditional Chinese medicine

Sal B: Salvianolic acid B

RA: Rosmarinic acid

HPLC: High-performance liquid chromatography

GO: Gene Ontology

COG: Clusters of Orthologous Groups of proteins

KO: $\quad$ KEGG Ortholog database

GRAS: Generally Recognized as Safe

ID: Identifier

KEGG: Kyoto Encyclopedia of Genes and Genomes

KAAS: KEGG Automatic Annotation Server
The authors have declared that no competing interests exist.

\section{Authors' Contributions}

Zhenqiao Song and Linlin Guo performed the analysis and wrote the paper; Tian Liu and Caicai Lin conducted the data analysis and prepared various figures; Xingfeng Li designed the study and provided samples; and Jianhua Wang contributed to paper preparation. All authors read and approved the paper. Zhenqiao Song and Linlin Guo contributed equally to this work and should be considered co-first authors.

\section{Acknowledgments}

This research was supported by the National Natural Science Foundation of China (81001603 and 81274012) and by the Natural Science Foundation of Shandong Province (ZR2015HM020).

\section{References}

[1] L. Zhou, Z. Zuo, and M. S. S. Chow, "Danshen: an overview of its chemistry, pharmacology, pharmacokinetics, and clinical use," Journal of Clinical Pharmacology, vol. 45, no. 12, pp. 1345-1359, 2005.

[2] Y. Ma, L. Yuan, B. Wu, X. Li, S. Chen, and S. Lu, "Genomewide identification and characterization of novel genes involved in terpenoid biosynthesis in Salvia miltiorrhiza," Journal of Experimental Botany, vol. 63, no. 7, pp. 2809-2823, 2012.

[3] Y. Lu and L. Y. Foo, "Polyphenolics of Salvia-a review," Phytochemistry, vol. 59, no. 2, pp. 117-140, 2002.

[4] Pharmacopoeia Commission of PRC, Ed., Pharmacopoeia of the People's Republic of China, Chemical Industry Press, Beijing, China, 2015.

[5] P. Hu, G.-A. Luo, Z. Zhao, and Z.-H. Jiang, "Quality assessment of radix Salviae Miltiorrhizae," Chemical and Pharmaceutical Bulletin, vol. 53, no. 5, pp. 481-486, 2005.

[6] G.-X. Zhong, P. Li, L.-J. Zeng, J. I. A. Guan, L. I. De-Qiang, and S.-P. Li, "Chemical characteristics of Salvia miltiorrhiza (Danshen) collected from different locations in China," Journal of Agricultural and Food Chemistry, vol. 57, no. 15, pp. 68796887, 2009. 
[7] H. J. Lee, K. H. Lee, K.-H. Park, and J.-H. Moon, "Large scale isolation and purification of Salvianolic acid B in high purity from roots of dansham (Salvia miltiorrhiza Bunge)," Food Science and Biotechnology, vol. 19, no. 2, pp. 497-502, 2010.

[8] Y. Xiao, S. Gao, P. Di, J. Chen, W. Chen, and L. Zhang, "Methyl jasmonate dramatically enhances the accumulation of phenolic acids in Salvia miltiorrhiza hairy root cultures," Physiologia Plantarum, vol. 137, no. 1, pp. 1-9, 2009.

[9] H. Yamamoto, P. Zhao, K. Yazaki, and K. Inoue, "Regulation of lithospermic acid B and shikonin production in Lithospermum erythrorhizon cell suspension cultures," Chemical and Pharmaceutical Bulletin, vol. 50, no. 8, pp. 1086-1090, 2002.

[10] M. Petersen, E. Häusler, B. Karwatzki, and J. Meinhard, "Proposed biosynthetic pathway for rosmarinic acid in cell cultures of Coleus blumei Benth," Planta, vol. 189, no. 1, pp. 10-14, 1993.

[11] B. Huang, B. Yi, Y. Duan et al., "Characterization and expression profiling of tyrosine aminotransferase gene from Salvia miltiorrhiza (Dan-shen) in rosmarinic acid biosynthesis pathway," Molecular Biology Reports, vol. 35, no. 4, pp. 601-612, 2008.

[12] J. Song and Z. Wang, "Molecular cloning, expression and characterization of a phenylalanine ammonia-lyase gene (SmPAL1) from Salvia miltiorrhiza," Molecular Biology Reports, vol. 36, no. 5, pp. 939-952, 2009.

[13] G. Cui, L. Huang, X. Tang, and J. Zhao, "Candidate genes involved in tanshinone biosynthesis in hairy roots of Salvia miltiorrhiza revealed by cDNA microarray," Molecular Biology Reports, vol. 38, no. 4, pp. 2471-2478, 2011.

[14] Y. P. Yan, Z. Z. Wang, W. Tian, Z. M. Dong, and D. F. Spencer, "Generation and analysis of expressed sequence tags from the medicinal plant Salvia miltiorrhiza," Science China Life Sciences, vol. 53, no. 2, pp. 273-285, 2010.

[15] Y. Li, C. Sun, H.-M. Luo, X.-W. Li, Y.-Y. Niu, and S.-L. Chen, "Transcriptome characterization for Salvia miltiorrhiza using 454 GS FLX," Acta Pharmaceutica Sinica, vol. 45, no. 4, pp. 524$529,2010$.

[16] W. P. Hua, Y. Zhang, J. Song, L. J. Zhao, and Z. Z. Wang, "De novo transcriptome sequencing in Salvia miltiorrhiza to identify genes involved in the biosynthesis of active ingredients," Genomics, vol. 98, no. 4, pp. 272-279, 2011.

[17] L. Yang, G. Ding, H. Lin et al., "Transcriptome analysis of medicinal plant Salvia miltiorrhiza and identification of genes related to tanshinone biosynthesis," PLoS ONE, vol. 8, no. 11, Article ID e80464, 2013.

[18] W. Gao, H.-X. Sun, H. Xiao et al., "Combining metabolomics and transcriptomics to characterize tanshinone biosynthesis in Salvia miltiorrhiza," BMC Genomics, vol. 15, article 73, 2014.

[19] B. Wang, W. Sun, Q. Li et al., "Genome-wide identification of phenolic acid biosynthetic genes in Salvia miltiorrhiza," Planta, vol. 241, no. 3, pp. 711-725, 2014.

[20] Y. Yu, J. Wei, X. Zhang et al., "SNP discovery in the transcriptome of white pacific shrimp Litopenaeus vannamei by next generation sequencing," PLoS ONE, vol. 9, no. 1, Article ID e87218, 2014.

[21] M. G. Grabherr, B. J. Haas, M. Yassour et al., "Full-length transcriptome assembly from RNA-Seq data without a reference genome," Nature Biotechnology, vol. 29, no. 7, pp. 644-652, 2011.

[22] I. Korf, M. Yandell, and J. Bedell, BLAST: An Essential Guide to the Basic Alignment Search Tool, O'Reilly and Associates, Sebastopol, Calif, USA, 2003.

[23] S. Götz, J. M. García-Gómez, J. Terol et al., "High-throughput functional annotation and data mining with the Blast2GO suite," Nucleic Acids Research, vol. 36, no. 10, pp. 3420-3435, 2008.

[24] M. Kanehisa, S. Goto, Y. Sato, M. Furumichi, and M. Tanabe, "KEGG for integration and interpretation of large-scale molecular data sets," Nucleic Acids Research, vol. 40, no. 1, pp. D109D114, 2012.

[25] B. Li and C. N. Dewey, "RSEM: accurate transcript quantification from RNA-Seq data with or without a reference genome," BMC Bioinformatics, vol. 12, article no. 323, 2011.

[26] A. Mortazavi, B. A. Williams, K. McCue, L. Schaeffer, and B. Wold, "Mapping and quantifying mammalian transcriptomes by RNA-Seq," Nature Methods, vol. 5, no. 7, pp. 621-628, 2008.

[27] S. Anders and W. Huber, "Differential expression analysis for sequence count data," Genome Biology, vol. 11, no. 10, article R106, 2010.

[28] M. D. Young, M. J. Wakefield, G. K. Smyth, and A. Oshlack, "Gene ontology analysis for RNA-seq: accounting for selection bias," Genome Biology, vol. 11, no. 2, article no. r14, 2010.

[29] X. Mao, T. Cai, J. G. Olyarchuk, and L. Wei, "Automated genome annotation and pathway identification using the KEGG Orthology (KO) as a controlled vocabulary," Bioinformatics, vol. 21, no. 19, pp. 3787-3793, 2005.

[30] Y. Yang, S. Hou, G. Cui, S. Chen, J. Wei, and L. Huang, "Characterization of reference genes for quantitative real-time PCR analysis in various tissues of Salvia miltiorrhiza," Molecular Biology Reports, vol. 37, no. 1, pp. 507-513, 2010.

[31] N. J. Bate, J. Orr, W. Ni et al., "Quantitative relationship between phenylalanine ammonia-lyase levels and phenylpropanoid accumulation in transgenic tobacco identifies a ratedetermining step in natural product synthesis," Proceedings of the National Academy of Sciences of the United States of America, vol. 91, no. 16, pp. 7608-7612, 1994.

[32] Y. Zhang, Y.-P. Yan, and Z.-Z. Wang, “The Arabidopsis PAP1 transcription factor plays an important role in the enrichment of phenolic acids in Salvia miltiorrhiza," Journal of Agricultural and Food Chemistry, vol. 58, no. 23, pp. 12168-12175, 2010.

[33] K. H. Kim, V. Janiak, and M. Petersen, "Purification, cloning and functional expression of hydroxyphenylpyruvate reductase involved in rosmarinic acid biosynthesis in cell cultures of Coleus blumei," Plant Molecular Biology, vol. 54, no. 3, pp. 311323, 2004.

[34] M. Sander and M. Petersen, "Distinct substrate specificities and unusual substrate flexibilities of two hydroxycinnamoyltransferases, rosmarinic acid synthase and hydroxycinnamoylCoA:shikimate hydroxycinnamoyl-transferase, from Coleus blumei Benth," Planta, vol. 233, no. 6, pp. 1157-1171, 2011.

[35] K. Meyer, A. M. Shirley, J. C. Cusumano, D. A. Bell-Lelong, and C. Chapple, "Lignin monomer composition is determined by the expression of a cytochrome P450-dependent monooxygenase in Arabidopsis," Proceedings of the National Academy of Sciences of the United States of America, vol. 95, no. 12, pp. 66196623, 1998.

[36] J.-H. Kim, D. H. Yang, J.-S. Kim et al., "Cloning, characterization, and expression of two cDNA clones for a rice ferulate-5hydroxylase gene, a cytochrome P450-dependent monooxygenase," Journal of Plant Biology, vol. 49, no. 3, pp. 200-204, 2006.

[37] A. Eudes, B. Pollet, R. Sibout et al., "Evidence for a role of AtCAD 1 in lignification of elongating stems of Arabidopsis thaliana," Planta, vol. 225, no. 1, pp. 23-39, 2006.

[38] W. Boerjan, J. Ralph, and M. Baucher, "Lignin biosynthesis," Annual Review of Plant Biology, vol. 54, pp. 519-546, 2003. 
[39] X. Q. Jin, Z. W. Chen, R. H. Tan, S. J. Zhao, and Z. B. Hu, "Isolation and functional analysis of 4-coumarate: coenzyme A ligase gene promoters from Salvia miltiorrhiza," Biologia Plantarum, vol. 56, no. 2, pp. 261-268, 2012.

[40] Z. Wang, L. Cui, C. Chen, X. Liu, Y. Yan, and Z. Wang, "Downregulation of cinnamoyl $\mathrm{CoA}$ reductase affects lignin and phenolic acids biosynthesis in Salvia miltiorrhiza bunge," Plant Molecular Biology Reporter, vol. 30, no. 5, pp. 1229-1236, 2012.

[41] Y. Zhang, Y.-P. Yan, Y.-C. Wu et al., "Pathway engineering for phenolic acid accumulations in Salvia miltiorrhiza by combinational genetic manipulation," Metabolic Engineering, vol. 21, pp. 71-80, 2014. 

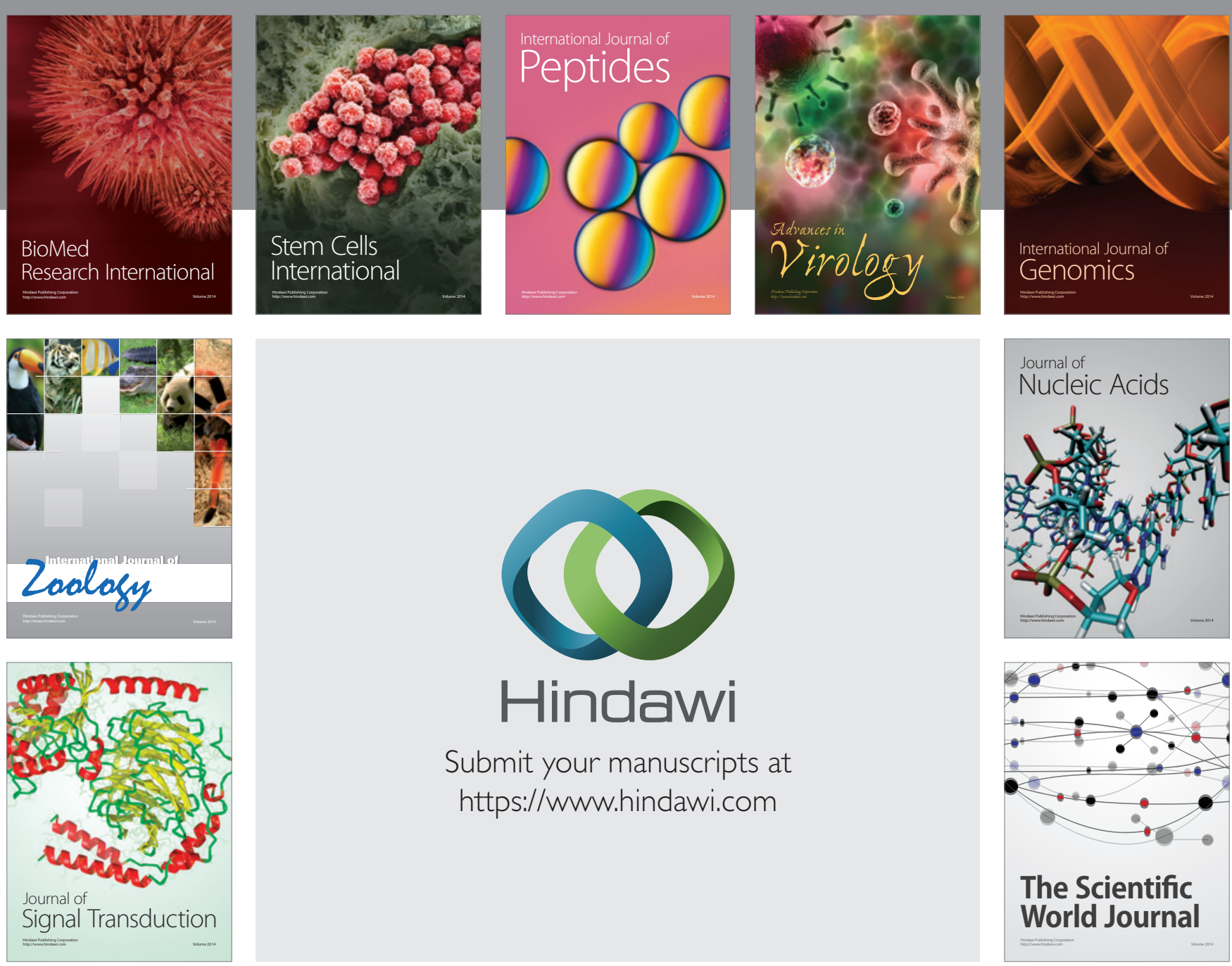

Submit your manuscripts at

https://www.hindawi.com
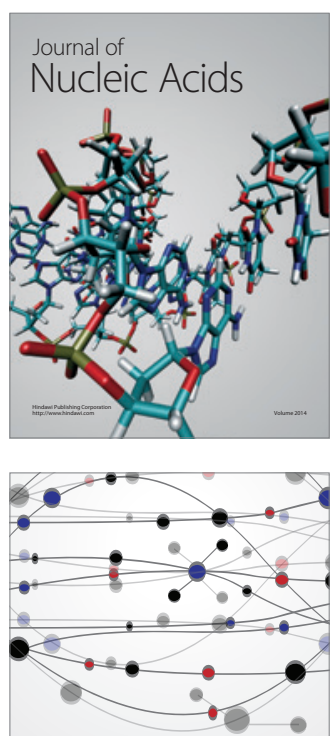

The Scientific World Journal
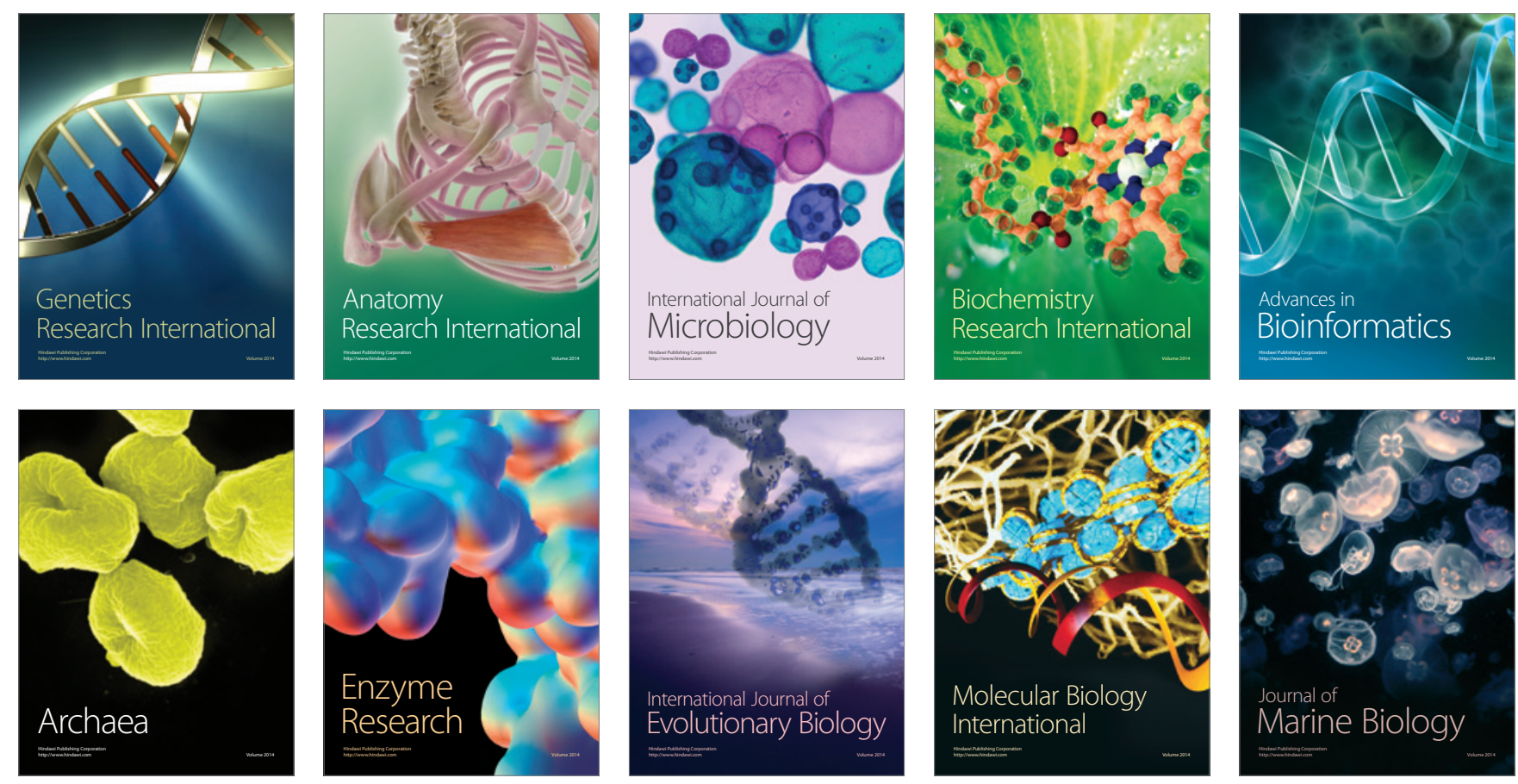